\title{
OPTIMAL LOW-DIMENSIONAL DYNAMICAL APPROXIMATIONS
}

\author{
BY \\ L. SIROVICH, B. W. KNIGHT * , AND J. D. RODRIGUEZ \\ Brown University
}

\begin{abstract}
We present a method for determining optimal coordinates for the representation of an inertial manifold of a dynamical system. The condition of optimality is precisely defined and is shown to lead to a unique basis system. The method is applied to the Neumann and Dirichlet problems for the Ginzburg-Landau equation. Substantial reduction in the size of the dynamical system, without loss of accuracy is obtained from the method.
\end{abstract}

1. Introduction. In many applications one is confronted with dynamics in a representational space which is large, perhaps infinite. Often this is accompanied by the belief that a description involving a smaller space is possible. This belief is based on theoretical or experimental results of various kinds. The most elementary of these is the fact that simple phenomena should be reducible to simple systems. For example, limit cycle (or two-torus) behavior, even in an infinite space, as can occur in RayleighBénard convection or Taylor-Couette flow, is representable by dynamics in a two- (or three-) dimensional phase space. Otherwise stated, if the phenomenon in question is first represented in some arbitrary way, then if the motion shows limit cycle behavior all dependent variables are representable as functions of just one distinguished dependent variable (two if it is two-torus motion).

This picture may be regarded as a specialized or limiting form of the masterslave formulation, which appears in a number of applications in which multiple timescales appear [1-3]. Typically one has a slow, evolving time scale describing the salient features of the system, and a fast transient (or oscillating) time scale. Simplification of the system is then obtained as a consequence of the elimination of the fast scales. Perhaps the classic example, and triumph, of this approach is the Chapman-Enskog procedure which leads from the Boltzmann equation, governing the molecular distribution, $f(x, \boldsymbol{\xi}, t)$, function, to the Navier-Stokes equation (in which only five moments with respect to the molecular velocity $\boldsymbol{\xi}$, of $f$, appear viz., $\rho, \mathbf{u}, T)[4,5]$.

The idea of attractors of dissipative systems may be relevant to such deliberations. Many dissipative systems, especially those related to fluid mechanics, show a tendency for the phase point to dwell on a relatively low-dimensional attractor,

Received September 7, 1989.

${ }^{*}$ Rockefeller University, New York. NY.

(C)1990 Brown University 
usually of fractional dimension. Perhpas the most famous example is that of the Lorenz system (itself a model of Benard convection)[6] in which case the equations are represented in a three-dimensional phase space and for which the attractor, at some reference parameter values, has the generally accepted measure of 2.06 [7]. Another example is the Ginzburg-Landau (G-L) equation [8], which, being a partial differential equation, has an infinite-dimensional representational space, but over some well-defined range of parameters it has a maximal attractor dimension of 3.05. $[9,10]$

In previous papers $[10,11,12]$ we have approached the problem of finding a finite approximation to such problems through the use of the Karhunen-Loève (K-L) procedure [13]. In brief, this determines a basis system which is optimal in the sense that a maximal fraction of the energy of the flow is captured, on average, for any finite-dimensional approximation. The basis functions generated in this way are then used in a Galerkin procedure to generate a dynamical system. In geometrical terms, the Galerkin procedure projects the dependent variables and the equations onto a finite-dimensional space. Thus the goal is to find an optimal hyperplane in which the attractor resides to a good approximation. Further, since the K-L procedure can be viewed as a principal axis decomposition, the procedure can be viewed as encapsulating the attractor in an ellipsoid-which becomes flatter and flatter as the dimension of approximating hyperplane is increased.

One can therefore expect to improve on the Galerkin procedure by slaving the previously neglected variables to the retained variables. That is, by finding algebraic relations between the neglected variables and the retained variables. A mathematical framework for rigorously treating these ideas is contained in the study of inertial manifolds $[14,15,16]$. The inertial manifold by definition is a manifold to which solutions tend at an exponential rate. A caricature of the slaving approximation to the inertial manifold which we have described above is that it puts some bend in the encapsulating ellipsoid.

While the notion of an inertial manifold is intrinsic to a problem, its actual specification depends in an essential way on the representation. (As a trivial illustration we might consider an oscillator moving on a straight line in $N$-space. Unless one chooses the appropriate coordinate system the problem appears to involve $2 \mathrm{~N}$-dimensions.) We show in this paper how, by objective criteria, to optimally choose an approximate inertial manifold. This will be applied to two problems which arise from consideration of the G-L equation. In both instances a substantial reduction in the representational phase space is obtained.

2. An optimal basis set. For purposes of exposition we assume that the solution to the dynamical system

$$
\frac{d \mathbf{v}}{d t}=F[\mathbf{v}]
$$

is chaotic and is known to us in as much detail as we might wish. Next suppose that $\left\{\psi_{n}\right\}$ represents a complete orthonormal basis. It is unnecessary for us to distinguish between the finite or infinite case. In the latter case $\left\{\psi_{n}\right\}$ may be regarded as a basis 
set in a function space. At this stage the basis is unknown to us, and the object of the following deliberations is how to best determine the system, $\left\{\psi_{n}\right\}$.

Denote by $P_{N}$ the projection operator onto the first $N$ of these elements, e.g.,

$$
P_{N} \mathbf{v}=\mathbf{v}_{N}=\sum_{k=1}^{N}\left(\psi_{k}, \mathbf{v}\right) \psi_{k}
$$

where $(\cdot, \cdot)$ denotes the inner product under which $\left\{\psi_{n}\right\}$ are orthonormal. The ordering of the elements is important and will be discussed. To further fix matters we regard $N$ as prescribed, i.e., $N$ is fixed and the system $\left\{\psi_{n}\right\}$ is varied in the analysis below.

We write the complimentary vector as

$$
\mathbf{v}_{N}^{c}=\left(1-P_{N}\right) \mathbf{v}
$$

and therefore

$$
\mathbf{v}=\mathbf{v}_{N}+\mathbf{v}_{N}^{c}
$$

In this notation we split the dynamical system (1) by projecting the system point onto the projected space and complimentary spaces as follows,

$$
\begin{gathered}
P_{N} \frac{d \mathbf{v}}{d t}=\frac{d}{d t} \mathbf{v}_{N}=P_{N} \mathbf{F}\left(\mathbf{v}_{N}+\mathbf{v}_{N}^{c}\right)=\mathbf{Q}_{N}\left(\mathbf{v}_{N}+\mathbf{v}_{N}^{c}\right) \\
\left(1-P_{N}\right) \frac{d \mathbf{v}}{d t}=\frac{d}{d t} \mathbf{v}_{N}^{c}=\left(1-P_{N}\right) \mathbf{F}\left(\mathbf{v}_{N}+\mathbf{v}_{N}^{c}\right)=\mathbf{R}_{N}\left(\mathbf{v}_{N}+\mathbf{v}_{N}^{c}\right) .
\end{gathered}
$$

On inspecting these forms we might argue that if the set $\left\{\psi_{N}\right\}$ can be chosen so that $\mathbf{R}_{N}=0$, say asymptotically, and we can take asymptotic initial data (aged initial data) so that

$$
\left.\mathbf{v}_{N}^{c}(t)\right|_{t=0}=0
$$

then it would follow from $(6)$ that

$$
\mathbf{v}_{N}^{c}(t) \equiv 0
$$

and hence

$$
\frac{d \mathbf{v}_{N}}{d t} \equiv \mathbf{Q}_{N}\left(\mathbf{v}_{N}\right)
$$

The last system is just what would be obtained from a Galerkin procedure.

In order to implement the above procedure we write

$$
\|\mathbf{v}\|^{2}=\left\|\mathbf{v}_{N}\right\|^{2}+\left\|\mathbf{v}_{N}^{c}\right\|^{2}
$$

and then search for the basis set which minimizes $\left\|\mathbf{v}_{N}^{c}\right\|^{2}$ (alternately, maximizes $\left.\left\|v_{N}\right\|^{2}\right)$. Since $\left\|\mathbf{v}_{N}^{c}\right\|^{2}$ is time dependent we instead minimize its ensemble average

$$
\varepsilon_{N}^{c}=\left\langle\left\|\mathbf{v}_{N}^{c}\right\|^{2}\right\rangle
$$

Or if we assume ergodicity, as we will, the time average of $\left\|\mathbf{v}_{N}^{c}\right\|^{2}$ is to be minimized. The solution to this problem is furnished by the K-L procedure [13]. Since the discussion of the next section parallels the K-L procedure and since this has been presented previously $[10,17,18]$, we do not pursue it further. 
3. An ideal inertial manifold basis. A natural extension, and hopefully an improvement, of the above approach lies in retaining the effect of the neglected variables $\mathbf{v}_{N}^{c}$. The essence of the idea is contained in the slaved variable approach (see [3] and references mentioned there). Instead of setting $\mathbf{v}_{N}^{c}=0$ we solve

$$
\mathbf{R}_{N}\left(\mathbf{v}_{N}+\mathbf{v}_{N}^{c}\right)=0
$$

for

$$
\mathbf{v}_{N}^{c}=\mathbf{S}\left(\mathbf{v}_{N^{N}}\right)
$$

substitute into (5)

$$
\frac{d}{d t} \mathbf{v}_{N}=\mathbf{Q}_{N}\left(\mathbf{v}_{N}+\mathbf{S}\left(v_{N}\right)\right)
$$

and solve this system. Thus the sets $\mathbf{v}_{N}^{c}$ are slaved in their time dependence to $\mathbf{v}_{N}$. This approach has been suggested as an approximation to the inertial manifold of the underlying system by Foias et al. [19] and Titi [20] for the Navier-Stokes equations.

If we examine the argument leading to the approximate inertial manifold, we observe that the objective of the procedure changes character as we pass from the Galerkin formulation to the system (14). For, to arrive at (9) we obtained a parametrization based on minimizing the energy error (11). However, in going to obtain (14) we first need to solve (12). But this implies that our principal objective should be the minimization of $\left\|\mathbf{R}_{N}\right\|$. Or, what is equivalent, to minimize in some sense $\left\|\dot{\mathbf{v}}_{N}^{c}\right\|^{2}$.

To develop this notion into a criterion, consider

$$
\|\dot{\mathbf{v}}\|^{2}=\left\|\dot{\mathbf{v}}_{N}\right\|^{2}+\left\|\dot{\mathbf{v}}_{N}^{c}\right\|^{2},
$$

where the norm is based on the inner product. Thus an alternate viewpoint is that we wish to maximize $\left\|\dot{\mathbf{v}}_{N}\right\|^{2}$. This quantity will vary in time and we therefore attempt instead to maximize its time average. Equivalently, under the assumption of ergodicity, we wish to maximize the ensemble averaged form,

$$
\dot{E}_{N}=\left\langle\left(\dot{\mathbf{v}}_{N}, \dot{\mathbf{v}}_{N}\right)\right\rangle \text {, }
$$

for arbitrary $N$.

Strictly speaking this is not the standard K-L procedure, but the analysis is similar and we outline the steps. (In the usual form of the K-L procedure $\left\langle\mathbf{v}_{N}, \mathbf{v}_{N}\right\rangle$ is maximized.) For this purpose we form, what might be termed, the acceleration covariance,

$$
\mathbf{L}=\langle\dot{\mathbf{v}} \otimes \dot{\mathbf{v}}\rangle,
$$

where $\otimes$ represents the outer product. The solution to the optimization problem is then given by the eigenvalue problem [17]

$$
\mathbf{L} \phi=\lambda \phi \text {. }
$$

Both (17) and (18) require some further explanation.

We denote by

$$
\left\{\dot{\mathbf{v}}\left(t_{n}\right)\right\}=\left\{\dot{\mathbf{v}}^{(n)}\right\}
$$


an ensemble (sufficiently large, say $M$ members) of uniformly (time) sampled states of the solution of (1). If (1) represents a finite system of ordinary differential equations in the components $v_{i}$ then

$$
L_{i j}=\left\langle\dot{v}_{i} \dot{v}_{j}\right\rangle=\frac{1}{M} \sum_{n}^{M} \dot{v}_{i}^{(n)} \dot{v}_{j}^{(n)}
$$

is a symmetric matrix and the orthonormal set $\left\{\phi_{k}\right\}$ represents the eigenvectors of $\mathbf{L},(14)$. On the other hand, if (1) represents a system of $P$ partial differential equations then we first obtain the ensemble

$$
\left\{\dot{v}_{i}\left(\mathbf{x}, t_{n}\right)\right\}=\left\{\dot{v}_{i}^{(n)}(\mathbf{x})\right\} ; \quad 1 \leq n \leq M ; 1 \leq i \leq P ;
$$

and then form the two point covariance matrix,

$$
L_{i j}\left(\mathbf{x}, \mathbf{x}^{\prime}\right)=\left\langle\dot{v}_{i}(\mathbf{x}) \dot{v}_{j}\left(\mathbf{x}^{\prime}\right)\right\rangle=\frac{1}{M} \sum_{n=1}^{M} \dot{v}_{i}^{(n)}(\mathbf{x}) \dot{v}_{j}^{(n)}\left(\mathbf{x}^{\prime}\right) .
$$

Then, by (18), we mean

$$
\int_{V} \mathbf{L}\left(\mathbf{x}, \mathbf{x}^{\prime}\right) \phi\left(\mathbf{x}^{\prime}\right) d \mathbf{x}^{\prime}=\lambda \phi(\mathbf{x}),
$$

when $V$ is the domain of the problem.

In any case the operator $\mathbf{L}$ is easily seen to be hermitian, nonnegative, and in particular instances can be proven to be square integrable. For example, for the G-L equation which we use as an illustration, Lagrange stability has been demonstrated [21], from which square integrability follows. Thus Mercer's theorem [22] assures us that $\left\{\phi_{k}\right\}$ form a complete orthonormal system. The K-L theorem [13] then states that

$$
\dot{\mathbf{v}}=\sum b_{n}(t) \phi_{n}, \quad b_{n}=\left(\phi_{n}, \dot{\mathbf{v}}\right)
$$

almost everywhere. It also follows that the coefficients, $\left\{b_{n}\right\}$, are statistically orthogonal,

$$
\left\langle b_{n} b_{m}\right\rangle=\mu_{n} \delta_{n m} .
$$

In previous treatments $[10,11,12]$ we have used the K-L procedure by forming the covariance matrix,

$$
\mathbf{K}=\langle\mathbf{v} \otimes \mathbf{v}\rangle,
$$

and then generating the corresponding eigenfunctions,

$$
\mathbf{K} \psi_{n}=\lambda_{n} \psi_{n} .
$$

The state variable is then expanded

$$
\mathbf{v}=\sum a_{n}(t) \boldsymbol{\psi}_{n}, \quad a_{n}=\left(\boldsymbol{\psi}_{n}, \mathbf{v}\right),
$$

and the coefficients $\left\{a_{n}\right\}$ are statistically orthogonal

$$
\left\langle a_{n} a_{m}\right\rangle=\lambda_{n} \delta_{n m} \text {. }
$$

To relate the two sets, $\left\{\phi_{n}\right\}$ and $\left\{\psi_{n}\right\}$, we form the symmetric nonnegative matrix,

$$
M_{n m}=\left\langle\dot{a}_{n} \dot{a}_{m}\right\rangle
$$


Then if $\left\{\ell_{n}\right\}$ denotes the components of an eigenvector of $\mathbf{M}$, one easily verifies that

$$
\phi=\sum \ell_{n} \psi_{n}
$$

is an eigenfunction of $L$.

It may be useful to close this section by summarizing the two different approaches, by once again contrasting their underlying philosophies.

In the standard K-L procedure we are led to the system $\left\{\boldsymbol{\psi}_{n}\right\}$, which are eigenfunctions of the covariance $\mathbf{K},(26)$. A finite truncation of $\mathbf{v}, \mathbf{v}_{N}$, is identified with the coefficients, (28), and we write

$$
\mathbf{v}_{N} \leftrightarrow \mathbf{a}=\left(a_{1}, \ldots, a_{N}\right),
$$

and similarly

$$
\mathbf{v}_{N}^{c} \leftrightarrow\left(a_{N+1}, \ldots\right) .
$$

The corresponding dynamical system may be written formally as

$$
\left.\begin{array}{rl}
\frac{d}{d t} \mathbf{a} & =\mathscr{P}\left(\mathbf{a}, \mathbf{a}^{c}\right) \\
\frac{d}{d t} \mathbf{a}^{c} & =\mathscr{S}^{c}\left(\mathbf{a}, \mathbf{a}^{c}\right) .
\end{array}\right\}
$$

In a similar fashion we consider the eigenfunctions, $\left\{\phi_{n}\right\}$, of the acceleration covariance $\mathbf{L},(17)$. The coefficients are now given by (24) and correspondingly we make the associations

$$
\begin{gathered}
\dot{\mathbf{v}}_{N} \leftrightarrow \mathbf{b}=\left(b_{1}, \ldots, b_{N}\right), \\
\dot{\mathbf{v}}_{N}^{c} \leftrightarrow \mathbf{b}^{c}=\left(b_{N+1}, \ldots\right) .
\end{gathered}
$$

We are then led to the dynamical system

$$
\left.\begin{array}{rl}
\frac{d \mathbf{b}}{d t} & =T\left(\mathbf{b}, \mathbf{b}^{c}\right) \\
\frac{d \mathbf{b}^{c}}{d t} & =T^{c}\left(\mathbf{b}, \mathbf{b}^{c}\right) .
\end{array}\right\}
$$

In the first instance, the split in variables has been made on the basis of energy. Thus $N$ is chosen so that for some criterion

$$
\left\langle\left\|\mathbf{a}^{c}\right\|^{2}\right\rangle<\varepsilon
$$

and under the approximation $\left\|\mathbf{a}^{c}\right\| \approx 0$ we are led to

$$
\frac{d \mathbf{a}}{d t}=\mathscr{P}(\mathbf{a}, 0),
$$

which is just the Galerkin procedure. It might be supposed that a better approximation results if instead of neglecting $\mathbf{a}^{c}$ we consider

$$
\left.\begin{array}{l}
\frac{d \mathbf{a}}{d t}=\mathscr{P}\left(\mathbf{a}, \mathbf{a}^{c}\right) \\
0=\mathcal{F}^{c}\left(\mathbf{a}, \mathbf{a}^{c}\right)
\end{array}\right\}
$$


where $a^{c}$ is solved for in the second relation and then substituted into the first. This is an example of the slaving principle, but it should be observed that the steps going into (36) did not start with the objective of using the slaving principle.

The method presented in this section starts with the premise that the slaving principle is to be applied. Thus in (33) the split in dependent variables is based on an accerleration energy, (16). The splitting index $N$ is chosen so that for some criterion $\varepsilon$

$$
\left\langle\| \dot{\mathbf{b}}^{c}||\right\rangle<\varepsilon
$$

From this it might be supposed that (33) can be approximated by

$$
\begin{gathered}
\frac{d \mathbf{b}}{d t}=\mathbf{T}\left(\mathbf{b}, \mathbf{b}^{c}\right) \\
0=\mathbf{T}^{c}\left(\mathbf{b}, \mathbf{b}^{c}\right) .
\end{gathered}
$$

In both cases (33) and (38) the algebraic relation is easily solved if the slaved variable $\left(\mathbf{a}^{c}\right.$ or $\left.\mathbf{b}^{c}\right)$ is supposed small. Titi [23] has suggested that an iteration based on this smallness assumption would converge very rapidly, since solutions converge exponentially to an inertial manifold.

4. Application to the Ginzburg-Landau equation. The G-L equation

$$
A_{t}-q^{2}\left(i+c_{0}\right) A_{x x}-\rho \dot{A}-(i-\rho) A|A|^{2}=0,
$$

has been well investigated numerically and theoretically and therefore serves as a useful example for exploring the ideas presented in the previous section. Two boundary value problems have been investigated in detail [11, 12], viz. the Dirichlet Problem:

$$
A(0)=A(\pi)=0 \text {, }
$$

and the Neumann Problem:

$$
\frac{\partial A(0)}{\partial x}=\frac{\partial A(\pi)}{\partial x}=0 .
$$

For all the numerical computations mentioned here, the parameters $c_{0}$ and $\rho$ have been fixed at $c_{0}=\rho=1 / 4$ and the active bifurcation parameter is $q$. A summary of behavior in the two cases is presented in Fig. 1.

The Galerkin procedure, associated with Eq. (39), has also been applied to each of these cases and we briefly summarize those results in each instance. For the Neumann problem in the range,

$$
.6 \leq q \leq 1.3 \text {, }
$$

the Lyapunov dimension of the attractor has a maximum of $d_{L}=3.047$ at $q=.95$. For this reason the K-L eigenfunctions were computed at this value of $q$ and from these a dynamical system was constructed according to (9) or (35). The criterion used to choose the cutoff index $N$ was that roughly $99 \%$ of the mean energy be captured. This led to a system of three complex equations [12]. The behavior of these six equations over the range (42) is virtually indistinguishable from exact integration $[10,12]$.

The Dirichlet problem was considered over the range [12]

$$
.01<q<1 \text {. }
$$


(a)

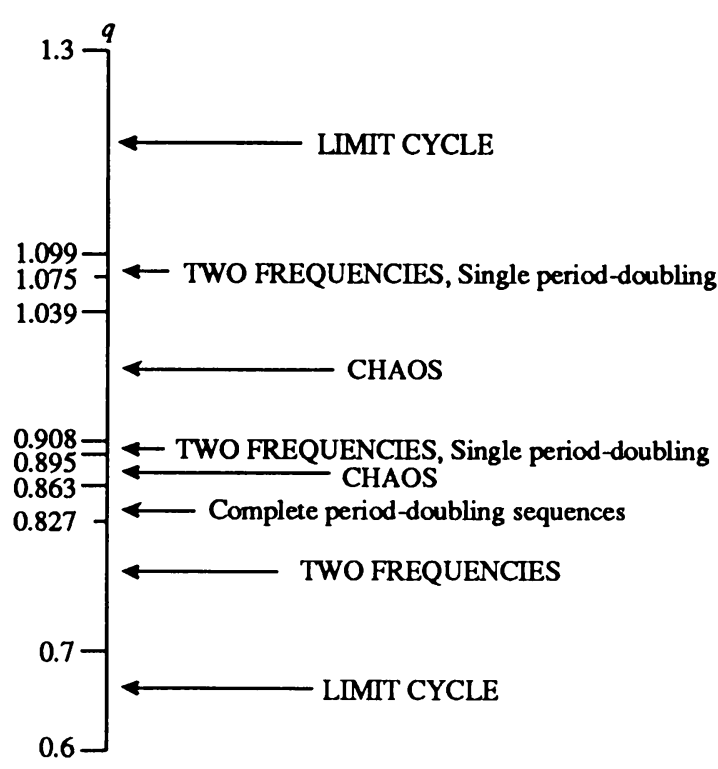

(b)

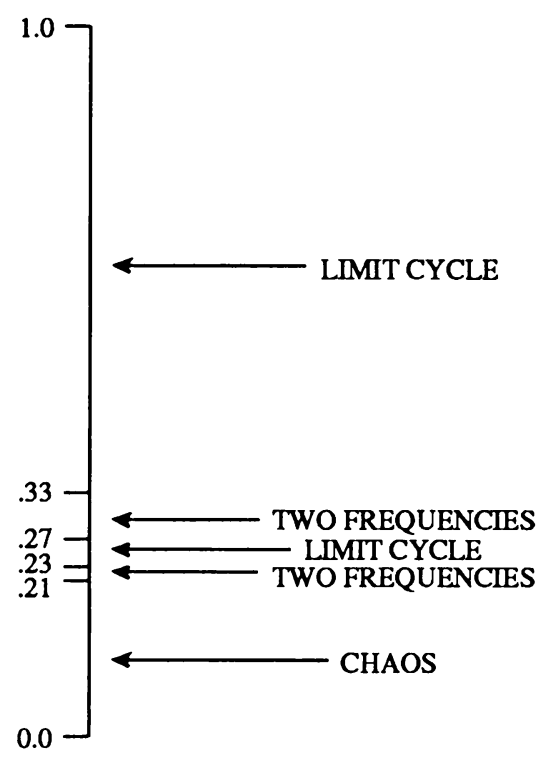

FIG. 1. Summary of behavior for (a) Neumann problem, (b) Dirichlet problem.

The Lyapunov dimension was computed over the range $.08<q<.3$ and found to increase monotonically. Since there existed no distinguished value of $q$ at which to compute the eigenfunctions, the value of $q=.14$ was arbitrarily chosen as the nominal value for the calculation. At this value of $q$ the Lyapunov dimension was found to be 9.1 . Again, using the $99 \%$ criterion, ten complex differential equations resulted. In the range, (43), the agreement between the exact and approximate systems was excellent [12].

Neumann problem. The database for this case at $q=.95$ was used to construct the acceleration covariance $\mathbf{L},(17)$, and from this its eigenfunctions. Table 1 contains the first three eigenvalues and contrasts these with those obtained for the covariance (26).

TABLE 1

\begin{tabular}{|c|c|c|}
\hline$n$ & $\lambda_{n}(\mathbf{K})$ & $\lambda_{n}(\mathbf{L})$ \\
\hline 1 & .8599 & .8970 \\
\hline 2 & .1380 & .1013 \\
\hline 3 & .0021 & .0019 \\
\hline
\end{tabular}

The eigenvalues here and later are normalized to be probabilities, i.e., their sum is unity.

Figure 2 contrasts the real parts of the first three eigenfunctions (the imaginary parts are small and are not shown [10]). As can be seen these functions are relatively close to one another in the two cases. 


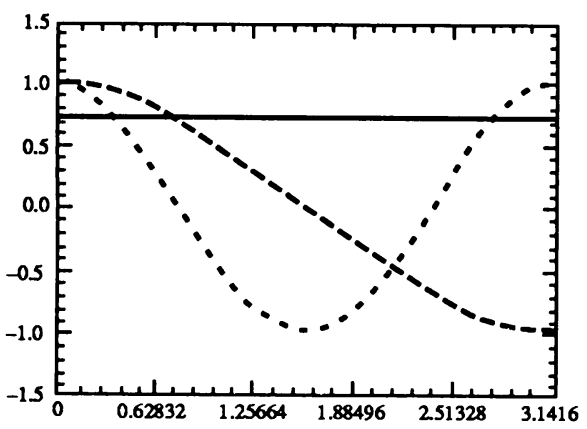

(a)

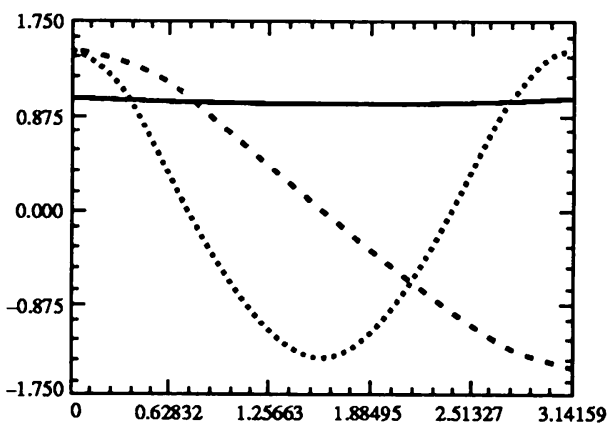

(b)

FIG. 2. Comparison of real parts of first three eigenfunctions for Neumann problem (a) K-L eigenfunctions, (b) Acceleration eigenfunctions.

The splitting of the dependent variables as given by (33) breaks these into dynamical, b, and algebraical, $\mathbf{b}^{c}$, variables. Since the latter are now being retained, we should expect the cutoff to be more forgiving in contrast with the Galerkin cutoff, i.e., a larger value of $\varepsilon$ should be tolerable. As mentioned earlier the maximal dimension of the attractor for the range (42) is $d_{L}=3.05$. From this it follows that the minimal number of equations we can hope for is four. The system was split into two complex dynamical equations corresponding to the first two eigenfunctions and an algebraic equation corresponding to the third eigenfunction. The resulting system is given in the Appendix. (In view of the smallness of the third coefficient, only the linearized form of algebraic equation is shown.)

The result of integrating the system given in the Appendix is as good as the six equation Galerkin approximation in depicting the phenomena in the range (42). All the transition points shown in Fig. 1 are obtained with the same accuracy. The same slaving procedure was also applied to the covariance formulation (32), and the result was essentially the same. I.e., with $N=2$, the system (32) accurately described the range (42). Perhaps, in view of the closeness of the two sets of eigenfunctions shown in Fig. 1, this is not surprising. This is doubtless due to the lack of spatial complexity in solutions of the Neumann problem in the range (42).

Dirichlet problem. As mentioned earlier, the nominal value of $q=.14$ was arbitrarily chosen, in this case, as the point at which to compute the eigenfunction basis of $\mathbf{L}$. Table 2 contrasts the eigenvalues of $\mathbf{K}$ and $\mathbf{L}$.

As was the case for Table 1 the two columns represent probabilities, but represent different measures. The square root of the eigenvalues in the first column represent $r m s$ amplitudes while the square root of the eigenvalues in the second column represent $r m s$ values of time derivatives.

In Fig. 3 we contrast the first four eigenfunctions of the $\mathbf{K}$ and $\mathbf{L}$ formulations. Unlike the Neumann case the two sets of eigenfunctions are now sharply different. The Galerkin approach, (35), based on the $K$-eigenfunctions, with $N=10$ (i.e., ten complex equations) gives excellent agreement with the exact numerical integration of 

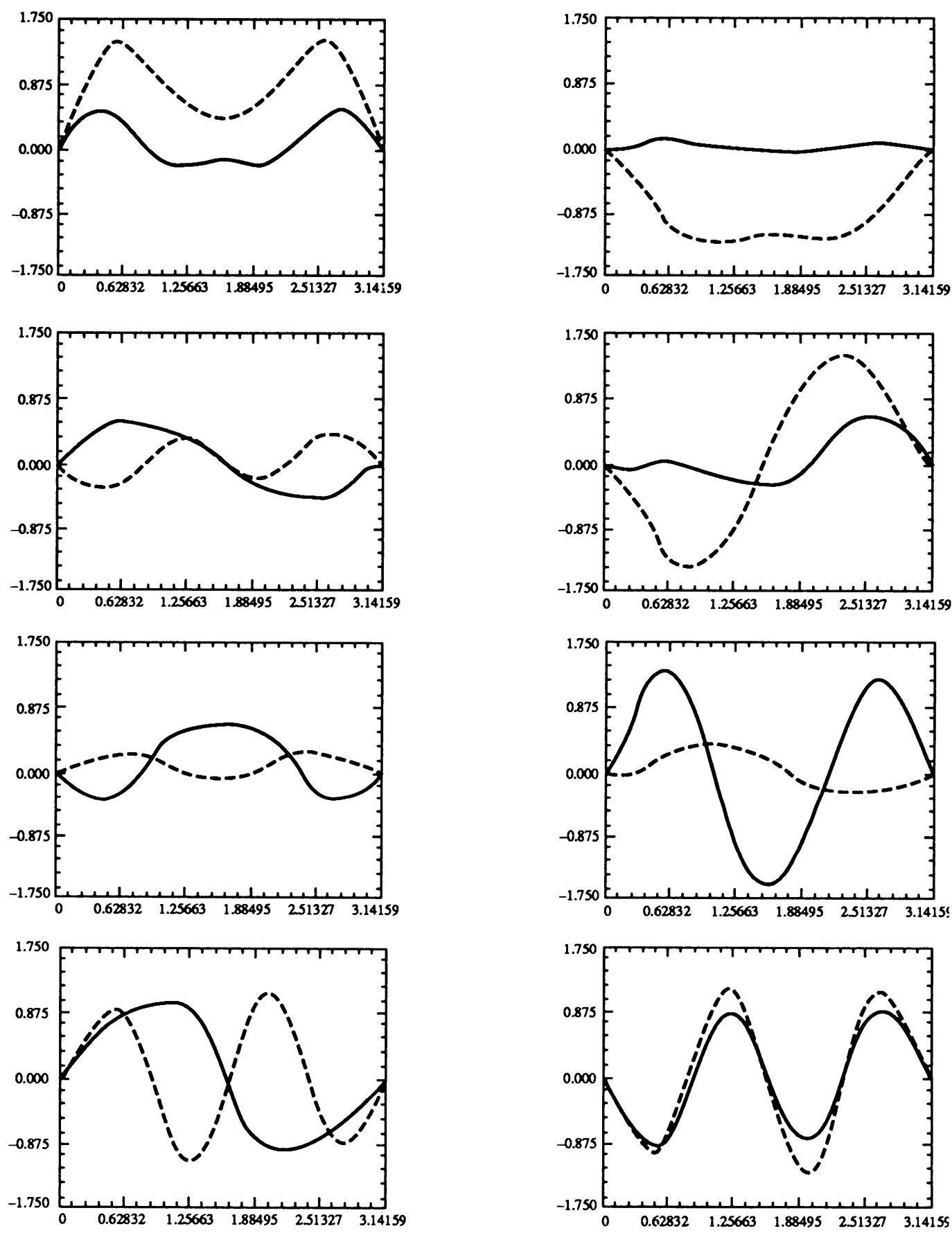

FIG. 3. Comparison of real (continuous and imaginary (dashed) parts of first four eigenfunctions for Dirichlet problem. (a) K-L eigenfunctions. (b) Acceleration eigenfunctions. 
TABLE 2

\begin{tabular}{|c|c|c|}
\hline$n$ & $\lambda_{n}(\mathbf{K})$ & $\lambda_{n}(\mathbf{L})$ \\
\hline 1 & .2602 & .3698 \\
2 & .1997 & .2178 \\
3 & .1870 & .1810 \\
4 & .1334 & .0964 \\
5 & .1110 & .0492 \\
6 & .0499 & .0243 \\
7 & .0290 & .0200 \\
8 & .0135 & .0137 \\
9 & .0075 & .0103 \\
10 & .0038 & .0065 \\
11 & .0022 & .0041 \\
\hline
\end{tabular}

(39) for the range $.1<q<1$ [11]. To examine the approach using an approximate inertial manifold we chose $N=6$ in (38).

Excellent agreement with the exact results, for $.1<q<1$ was obtained for (38). All transitions were accurately described with this system. (With $N=5$ spurious windows of relaminarization appeared.) A similar investigation was made using the formulation (36) based on the $K$-eigenfunctions. In this case we could not go below $N=7$. But the agreement was again good over the range $.1<q<1$ for this splitting. Thus the improvement of the $L$-formulation over the $K$-formulation is not dramatic in this instance.

We close by contrasting the 6-mode theory (approximate inertial manifold), the 10-mode (Galerkin procedure) and the exact theory (pseudospectral method [11]) in Tables 3 and 4. Table 3 indicates the attractor dimension as measured by the Lyapunov dimension. The exact theory leads to a growth in dimension which is inversely proportional to the bifurcation parameter, $q$. Therefore any finite-dimensional dynamical approximation must eventually underestimate this measure. For example, 10 -mode theory is seen to match the exact dimension quite well for $q=.14$ and $q=.1$ but (understandably) seriously underestimates the dimension at $q=.08$. On the other hand 6-mode theory consistently underestimates the Lyapunov dimension. It is of some interest to determine whether such a discrepancy is necessarily a flaw. Typical power spectra (not shown here) are extremely close in appearance in all instances. Since the transitions shown in Fig. 1 are well modeled by both 6and 10-mode theory we must go to other measures to distinguish the approximate formulations from the exact results. An important extensive measure of the motion, is the mean energy

$$
\langle E\rangle=\left\langle\frac{1}{\pi} \int_{0}^{\pi}|A|^{2} d x\right\rangle .
$$

The energy is compared in Table 4. We see that in spite of the significant differences in the attractor dimension the energy predicted by the model systems is reasonably accurate except at $q=.08$ for the 6-mode theory. In our calculations we used the linearized form of the algebraic slaved variable equation and hence a further improvement may result from a more exact calculation. 
To end on a note of optimism we observe that the case treated indicates that a reasonable statistical description of the dynamics can be obtained without matching the attractor dimension.

TABLE 3. Lyapunov dimension.

\begin{tabular}{|c|c|c|c|}
\hline$q=$ & .14 & .1 & .08 \\
\hline 6-mode & 8.41 & 8.67 & 8.91 \\
\hline 10-mode & 9.16 & 13.92 & 14.51 \\
\hline exact & 9.09 & 13.84 & 17.76 \\
\hline
\end{tabular}

TABLE 4. Energy.

\begin{tabular}{|c|c|c|c|}
\hline$q=$ & .14 & .1 & .08 \\
\hline 6-mode & .402 & .456 & .530 \\
\hline 10-mode & .402 & .453 & .471 \\
\hline exact & .394 & .415 & .425 \\
\hline
\end{tabular}

Acknowledgment. The work reported here was supported by DARPA-URI N0001486-K0754.

Appendix.

$$
\begin{aligned}
\dot{A}_{0}= & -q^{2}(.0020+.0081 i) A_{0} \\
& +q^{2}(.0587-.1764 i) A_{2}+.25 A_{0} \\
& +(-.2514+1.0058 i) A_{0}\left|A_{0}\right|^{2} \\
& +(-.01899+.07593 i) A_{0}^{2} A_{2}^{*} \\
& +(-.3012+1.0470 i) A_{1}^{2} A_{0}^{*} \\
& +(-.5286+2.114 i) A_{0}\left|A_{1}\right|^{2} \\
& +(-.2290+.7422 i) A_{1}^{2} A_{2}^{*} \\
& +(-.03795+.15186 i) A_{2}\left|A_{0}\right|^{2} \\
& +(-.2508+1.0015 i) A_{2}^{2} A_{0}^{*} \\
& +(-.3767+1.506 i) A_{2}\left|A_{1}\right|^{2} \\
& +(-.5009+2.0037 i) A_{0}\left|A_{2}\right|^{2} \\
& +(-.01832+.04283 i) A_{2}\left|A_{2}\right|^{2}
\end{aligned}
$$




$$
\begin{aligned}
\dot{A}_{1}= & -q^{2}(.2521+1.0084 i) A_{1}+.25 A_{1} \\
& +(-.2269+1.0655 i) A_{0}^{2} A_{1}^{*} \\
& +(-.5286+2.114 i) A_{1}\left|A_{0}\right|^{2} \\
& +(-.3916+1.566 i) A_{1}\left|A_{1}\right|^{2} \\
& +(-.1883+.7532 i) A_{1} A_{2} A_{0}^{*} \\
& +(-.1883+.7532 i) A_{1} A_{2}^{*} A_{0} \\
& +(-.1472+.7626 i) A_{0} A_{1}^{*} A_{2} \\
& +(-.2210+1.0347 i) A_{1}^{*} A_{2}^{2} \\
& +(-.5133+2.053 i) A_{1}\left|A_{1}\right|^{2}
\end{aligned}
$$

where $A_{2}$ satisfies

$$
\begin{aligned}
0= & -q^{2}(.1348+.1280 i) A_{0}+(-.5133+2.053 i) A_{2}\left|A_{1}\right|^{2} \\
& -q^{2}(1.005+4.020 i) A_{2}+.25 A_{2} \\
& +(-.01899+.07593 i) A_{0}\left|A_{0}\right|^{2} \\
& +(-.250+1.002 i) A_{0}^{2} A_{2}^{*} \\
& +(-.2290+.7422 i) A_{1}^{2} A_{0}^{*} \\
& +(-.3765+1.506 i) A_{0}\left|A_{1}\right|^{2} \\
& +(-.2919+1.0169 i) A_{1}^{2} A_{2}^{*} \\
& +(-.5009+2.004 i) A_{2}\left|A_{0}\right|^{2}
\end{aligned}
$$

\section{REFERENCES}

[1] J. Kevorkian and J. D. Cole, Perturbation Method in Applied Mathematics, Springer-Verlag, NY, 1981

[2] H. Haken, Synergetics, 3rd Edition, Springer-Verlag, NY, 1983

[3] N. G. van Kampen, Elimination of fast variables, Phys. Rep. 124, 69-160 (1985)

[4] S. Chapman and T. G. Cowling, The Mathematical Theory of Nonuniform Gases, Cambridge University Press, 1952

[5] C. Cercignani, The Boltzmann Equation and its Application, Springer-Verlag, NY, 1988

[6] E. N. Lorenz, Deterministic nonperiodic flow, J. Atmos. Sci. 30, 130 (1963)

[7] I. Shimada and T. Nagaskima, A numerical approach to the ergodic problem of dissipative dynamical systems, Prog. Theor. Phys. 61, 1605 (1979)

[8] V. L. Ginzburg and L. D. Landau, Zh. Eksp. Teor. Fiz. 20 (1950)

[9] L. Keefe, Dynamics of perturbed wavetrain solutions to the Ginzburg-Landau equation, Stud. Appl. Math. 73, 91-153 (1985)

[10] L. Sirovich and J. D. Rodriguez, Coherent structures and chaos: A model problem, Physics Letter A 120, $211(1987)$

[11] L. Sirovich, J. D. Rodriguez, and B. Knight, Two boundary value problems for the Ginzburg Landau equation, Physica D 43 (1990)

[12] J. D. Rodriguez and L. Sirovich, Low dimensional dynamics for the complex Ginzburg Landau equation, Physica D 43 (1990)

[13] R. B. Ash and M. F. Gardner, Topics in Stochastic Processes, Academic Press, NY, 1975

[14] C. Foias, G. R. Sella, and R. Temam, Inertial manifolds for nonlinear evolutionary equations, J. Differential Equations 73, 309-353 (1988) 
[15] J. Mallet-Paret and G. R. Sell, Inertial manifolds for reaction-diffiusion equations in higher space dimensions, J. Amer. Math. Soc., 805-866 (1988)

[16] P. Constantin, C. Foias, B. Nicolenko, and R. Temam, Integral Manifolds and Inertial Manifolds for Dissipative Partial Differential Equations, Applied Mathematical Sciences, Springer-Verlag. New York-Berlin, 1989

[17] L. Sirovich, Turbulence and the dynamics of coherent structures, Pt. I: Coherent Structures, Quart. Appl. Math. 45 (3), 561-571 (1987)

[18] L. Sirovich, Chaotic dynamics of coherent structures, Physica D 37, 126-145 (1989)

[19] D. Foias, O. P. Manley, and R. Temam, Sur l'interaction des petits et grands tourbillars dans les écoulements turbulents, C. R. Acad. Sci. Paris, Serie I, 305. 497-500 (1987)

[20] E. Titi, On approximate inertial manifolds to the Navier-Stokes equations, Math. Sci. Inst. Rep. (Cornell), 1989

[21] P. Newton and L. Sirovich, Instabilities in the Ginzburgh-Landau equation: Periodic solutions, Quart. Appl. Math. 44 (49), (1986)

[22] F. Riesz and B. Sz. Nagy, Functional Analysis, Ungar, N.Y., 1955

[23] E. Titi, private communication

[24] J. M. Ghiadaglia and B. Héron, Dimension of the attractors associated to the Ginzburg-Landau partial differential equation, Physica 28D, 282-304 (1987) 